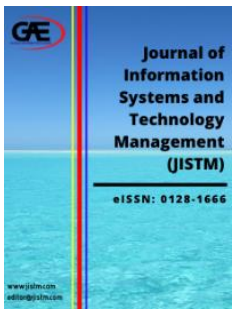

\author{
JOURNAL OF INFORMATION \\ SYSTEM AND TECHNOLOGY \\ MANAGEMENT (JISTM)

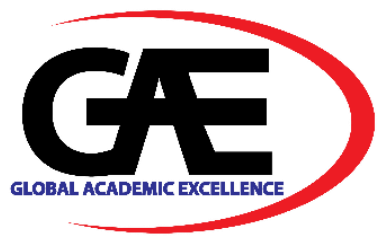

\title{
DEVELOPING A GOOD QUALITY CHATBOT
}

\author{
Nurul Muizzah Johari ${ }^{1 *}$, Puteri NE Nohuddin ${ }^{2}$ \\ 1 Institute of IR4.0, Universiti Kebangsaan Malaysia, Malaysia \\ Email: muizjohari91@gmail.com \\ 2 Institute of IR4.0, Universiti Kebangsaan Malaysia, Malaysia \\ Email: puteri.ivi@ukm.edu.my \\ Corresponding Author
}

\section{Article Info:}

Article history:

Received date: 10.06 .2021

Revised date: 15.07 .2021

Accepted date: 20.08 .2021

Published date: 01.09.2021

\section{To cite this document:}

Johari, N. M., \& Nohuddin, P. N. E. (2021). Developing a Good Quality Chatbot. Journal of Information System and Technology Management, 6 (22), 88-102.

DOI: $10.35631 /$ JISTM.622008

This work is licensed under CC BY 4.0

\begin{abstract}
:
Chatbots are commonly known nowadays as intelligent personal assistants which are designated to accomplish given tasks. The development of a good chatbot depends on the elements and features which then will be reflected in the performance and functionality of the application, user's experience as well as satisfaction in order to complete their task. Two methodologies are applied in this paper, which are preliminary study and literature survey. The outcomes were reflected into the development of a new chatbot based on the attributes gained from the observation. The aim of this paper is to investigate the possible quality attributes from past researches and to develop a platform that will subsequently be used to build a quality chatbot for the tourism domain.
\end{abstract}

Keywords:

Artificial Intelligence, Chatbot, Platform, Quality Attributes

\section{Introduction}

A chatbot is an application system which is able to mimics a human's natural language, created with the purpose of helping users in daily operations by automating designated tasks. Nowadays, they have become a part of customer service agents that help to escalate and assist users in completing their tasks, as an alternative to human agents (Folstad \& Skjuve, 2019). There are various sectors that have applied the system into their customer service practice, namely, education (Winkler, Rainer Söllner, \& Matthias (2018), Tamayo, Herrero, Martin, 
Navarro \& Tránchez (2020)), medical (Belfin, Shobana, Manilal, Mathew \& Babu (2019), Rarhi, Bhattacharya, Mishra \& Mandal (2017)), tourism (Ikpabi, Aslam \& Karjaluoto, 2019), and other significant sectors.

There are many selections of platforms available for developers to utilize in their development of chatbots. Patil (2020) shared a vast list of commonly used platforms to build chatbots. The common platforms are Botsify, Converse AI, Chatfuel, Flow XO, and other options with diverse functionalities.

The concerns with currently available chatbots are addressed by Barker (2021), such as the security level of a chatbot and its ability to understand the emotion and expression by users. The security of information and data shared by user through chatbots needs to be strong as it may content sensitive information. A lack of understanding of emotions could lead to misinterpretation, resulting in different outputs than what is expected by the user.

Another challenge detected by Gurwani (2018) is that the context used in chatbots is predefined to match human interaction patterns. However, these patterns may be change in some duration of time due to mixing of cultures and other possible triggering elements. Another challenge is user retention. Humans are expected to have a limited time span allocated to waiting on responses for their inquiries, where long waits may result in frustration. Thus, a chatbot with natural, creative, and fast responses is most favoured to cater for this issue. Another challenge is to test the bot itself, which may involve more than the targeted end users.

Studies on chatbot features have continuously and concurrently evolved with technology and the task demanding era. The basic guidelines to build the features of a chatbot can be started with the ISO 9126-1 Quality Model (ISO/IEC, 2001), which presented six (6) main categories; functionality, reliability, usability, efficiency, maintainability, as well as portability. Other researchers have expanded the list of attributes over time, which has resulted in a vast list of attributes to refer to.

The domain chosen as a baseline for the development of chatbot is tourism. In Malaysia, tourism has been recognised as one of the sectors that contributes the most to Malaysia's Gross Domestic Product (GDP). Most of the tourists rely on blogs, tourism websites as well as published advertisements, and local residents to promote the attractive places to visit. Thus, the development of this chatbot aims to simplify the effort needed by tourists to have instant information when travelling.

The aim of this paper is to review a series of literature on the history of chatbots, and the background as well as the optimum features recommended for a good quality chatbot. The following contents explain on the literature review, research methodology used, research outcome, and conclusion for this paper.

\section{Literature Review}

There will be three (3) points that be discussed in the literature review, which are, the history of chatbots, platforms available to build a chatbot, and quality attributes for a chatbot. 


\section{History of Chatbots}

The exploration of chatbots started in the 1950s when a Turing Test required the human judge to distinguish a conversation made between two parties; a human and a machine with ability to mimics a human's natural language. The outcomes proved the ability of the designed machine, whereby the judge was unable to determine which parties was the machine. From this successful event, the technology bloom witnessed the birth of ELIZA in 1960s, which used a simple pattern cross-checking technique and utilized the template to match the conversational style of a non-directional psychotherapist (Weizenbaum, 1966). This then continued with A.L.I.C.E (Artificial Intelligence Computer Entity) which was released in 1995; it functioned by using heuristic pattern matching rules from human input (Thompson, 2002). The evolution has continued with the current emergence of the intelligent personal assistants like Siri from Apple, Cortana from Microsoft, Alexa from Amazon, and Google Assistant from Google (Smutny \& Schreiberova, 2020). These applications are digital helpers that utilize machinelearning techniques to effectively solve daily tasks requested by user.

\section{Platform to Build Chatbots}

Multiple platforms can be found for the use of administrators and chatbot developers nowadays. These platforms help administrators to include more features by creating the flow, competencies of machine learning, integrations with APIs, as well as other significant functionalities (Aishwarya, Vijay \& Sachin, 2017). A summary of the top 7 favoured platforms used by chatbot developers was done by Patil (2020), as shown in TABLE 1 below.

Table 1: Favoured Platforms for Chatbot Development

\begin{tabular}{|c|c|}
\hline Platform & Description \\
\hline Botsify & $\begin{array}{l}\text { - Using drag and drop templates in chatbot creation. } \\
\text { - User-friendly features introduced such as plug-ins, Smart AI and also } \\
\text { Analytic Integration. }\end{array}$ \\
\hline FlowXO & $\begin{array}{l}\text { - Allows over } 100 \text { integrations and is equipped with an easy-to-use } \\
\text { visual editor. } \\
\text { - However, there are limitations on the number of conversations, which } \\
\text { then requires a subscription to bypass the limitations. }\end{array}$ \\
\hline Chatfuel & $\begin{array}{l}\text { - Allows user to include content cards and automatically share them } \\
\text { with the followers, collecting information from the conversation with } \\
\text { forms in Messenger Chats. Users are also able to make information } \\
\text { enquiries and use the buttons available within the bot. }\end{array}$ \\
\hline Bottr & $\begin{array}{l}\text { - Developing a personalised bot from the user's social media account } \\
\text { such as Facebook, LinkedIn, as well as Twitter. } \\
\text { - Provides an embed option to the user's website. }\end{array}$ \\
\hline Beep Boop & $\begin{array}{l}\text { - Provides an end-to-end experience when users embed the bot to their } \\
\text { own websites; adding data from other sources is also hassle-free. } \\
\text { - The bots interact with the user in real-time through chat and } \\
\text { messaging. }\end{array}$ \\
\hline Chattypeople & $\begin{array}{l}\text { - Makes use of pre-developed bots available for e-commerce, food } \\
\text { businesses, as well as customer support, with local conversation } \\
\text { experience. }\end{array}$ \\
\hline Motion.ai & $\begin{array}{l}\text { - The platform provided is edited by HubSpot and allows users or } \\
\text { developers to build a high-end bot without changing any code back- } \\
\text { end. }\end{array}$ \\
\hline
\end{tabular}


Volume 6 Issue 22 (September 2021) PP. 88-102 DOI: 10.35631/JISTM.622008

Platform

Description

- The baseline is from the constructed diagram of the conversation flow which than connects the bot to the messaging service used by the user.

Source: Top 30 Popular Platforms for Chatbot Development (Patil, 2020)

\section{Quality Attributes for a Good Chatbot}

A series of researches have been executed across the years to discover the quality attributes for developing a chatbot. The fundamental understanding starts with guidance provided in the software development process standards known as the ISO 9126 (ISO/ IEC, 2001). There are six (6) main characteristics representing the standards; functionality, reliability, usability, efficiency, maintainability, and portability.

- Functionality: the ability of any software to provide task-solving services to users that meets their needs and circumstances.

- Reliability: the ability to function in any condition or environment.

- Usability: easy to use; user-friendly, learnability, and simple handling.

- Efficiency: less time latency taken by the software to respond to the inputs and requests by users.

- Maintainability: easy to maintain, upgradable, easy to add new instruction or rule, and cost effective.

- Portability: ability to be ported across different settings.

Meerschman and Verkeyn (2019) have conducted a collective literature reading on quality attributes that a chatbot should be equipped with. The literature is dated from 1966 until 2019. There are 28 attributes that have been listed, with the aim to help the user and chatbot builders to evaluate any chatbot as widely as possible.

Table 2. Chatbot Quality Attributes (Adapted from Meerschman \& Verkeyn, 2019)

\begin{tabular}{|c|c|c|}
\hline Dimension & Quality Attributes & Reference \\
\hline \multirow[t]{6}{*}{ Functionality } & $\begin{array}{l}\text { Interpret commands } \\
\text { accurately }\end{array}$ & Morrisey and Kirakowski (2013) \\
\hline & Execute requested tasks & Explorative study \\
\hline & $\begin{array}{l}\text { Flexible in interpreting } \\
\text { knowledge }\end{array}$ & Cohen and Lane (2016) \\
\hline & Able to maintain a discussion & $\begin{array}{l}\text { Morrisey and Kirakowski (2013), } \\
\text { Kuligowska (2015) }\end{array}$ \\
\hline & Activation & $\begin{array}{l}\text { Hertzum et.a 1. (2002), Kuligowska } \\
\text { (2015) }\end{array}$ \\
\hline & $\begin{array}{l}\text { Number of services available } \\
\text { in the chatbot }\end{array}$ & Eeuwen (2017) \\
\hline \multirow[t]{4}{*}{ Trustworthiness } & $\begin{array}{l}\text { Containing dependable } \\
\text { information }\end{array}$ & Explorative study \\
\hline & $\begin{array}{l}\text { Possibility of rating the } \\
\text { chatbot }\end{array}$ & Kuligowska (2015) \\
\hline & $\begin{array}{l}\text { Contain a wide range of } \\
\text { knowledge }\end{array}$ & $\begin{array}{l}\text { Cohen and Lane (2016), } \\
\text { Kuligowska (2015) }\end{array}$ \\
\hline & $\begin{array}{l}\text { Robustness to unexpected } \\
\text { input }\end{array}$ & Kuligowska (2015) \\
\hline
\end{tabular}


Volume 6 Issue 22 (September 2021) PP. 88-102 DOI: 10.35631/JISTM.622008

\begin{tabular}{|c|c|c|}
\hline Dimension & Quality Attributes & Reference \\
\hline & Transparency & Hertzum et. al. (2002) \\
\hline \multirow[t]{2}{*}{ Safety/Intrusion } & Protect and respect privacy & Eeuwen (2017) \\
\hline & Save form intrusion & Duijst 92018) \\
\hline \multirow[t]{5}{*}{ Efficiency } & Ease of use & Candela (2018), Duijist (2018) \\
\hline & Quick replies vs free text & Duijst (2018) \\
\hline & Available at all times & Wang et. al. (2019) \\
\hline & Accessibility & Duijst (2018) \\
\hline & Need of an account & Explorative study \\
\hline Graphical & User-interface & Duijist (2018), Kuligowska (2015) \\
\hline Appearance & $\begin{array}{l}\text { Use of emojis and pictures / } \\
\text { gifs }\end{array}$ & Kuligowska (2015) \\
\hline \multirow[t]{4}{*}{ Humanity } & Realness of the chatbot & $\begin{array}{l}\text { Ramos (2017), Wallace (2003), } \\
\text { Weizenbaum (1966), Cohen (2005), } \\
\text { Whitby (1996) }\end{array}$ \\
\hline & $\begin{array}{l}\text { Create an enjoyable } \\
\text { interaction }\end{array}$ & Morrisey and Kirakowski (2013) \\
\hline & Convey personality & $\begin{array}{l}\text { Morrisey and Kirakowski (2013), } \\
\text { Kuligowska (2015) }\end{array}$ \\
\hline & Read and respond to moods & Beale and Creed (2009) \\
\hline \multirow[t]{2}{*}{ Empathy } & Personalization options & Kuligowska (2015) \\
\hline & Personalized suggestions & Explorative study \\
\hline \multirow[t]{2}{*}{ Responsiveness } & Responding immediately & Explorative study \\
\hline & Productivity & Rieke (2018), Candela (2018) \\
\hline
\end{tabular}

\section{Research Methodology}

This section will elaborate on the methodology used as well as the results gained once the test is completed. The aim of this paper is to develop a chatbot based on the good quality attributes gained from the literature survey done on the subject of interest. The preliminary study is conducted beforehand to gain a primary understanding of the user towards their experience while using chatbots that are currently available in Malaysia.

There are two stages involved, namely, pre-development and development. During the predevelopment stage, a set of structured questionnaires are shared to selected participants using Google Forms. The aim is to investigate the desired features and users' perceptions towards existing chatbots. Once all the data is gathered, it will be analysed and utilized in the next stage, the development of chatbot. Figure 1 below shows the summary of the flow for predevelopment stage. 


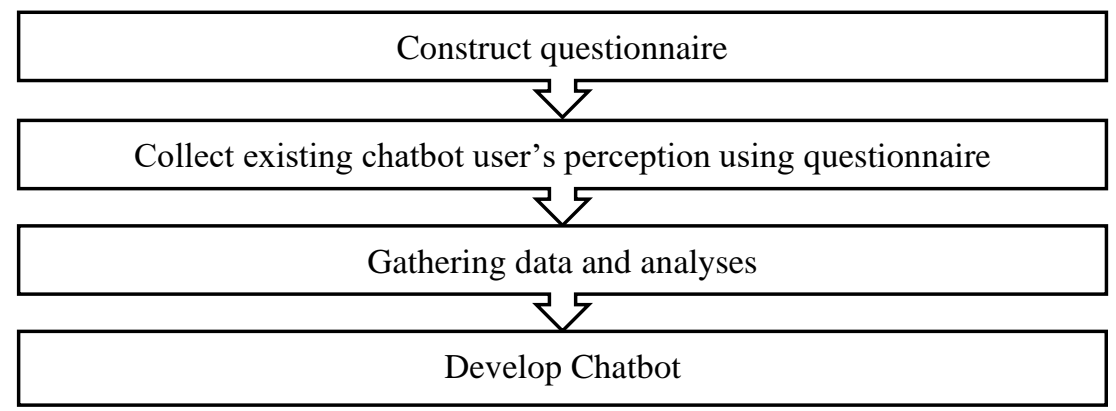

Figure 1:Pre-Development Stage

The second stage, the development of the chatbot, starts based on the outcome from the first stage and the literature survey. The flow is explained in Figure 2. Several intents are created as a baseline to train and prepare the bot before the actual conversation with end-user. A platform has been chosen to be used in the development stage of the chatbot; FlowXO. This platform allows any developer to train and add intent to the bot's knowledge simultaneously. The changes concurrently occur at the end-user's view. The platform has been integrated to Facebook Messenger, which provides accessibility and familiar feels to the functionality of the application to the user.

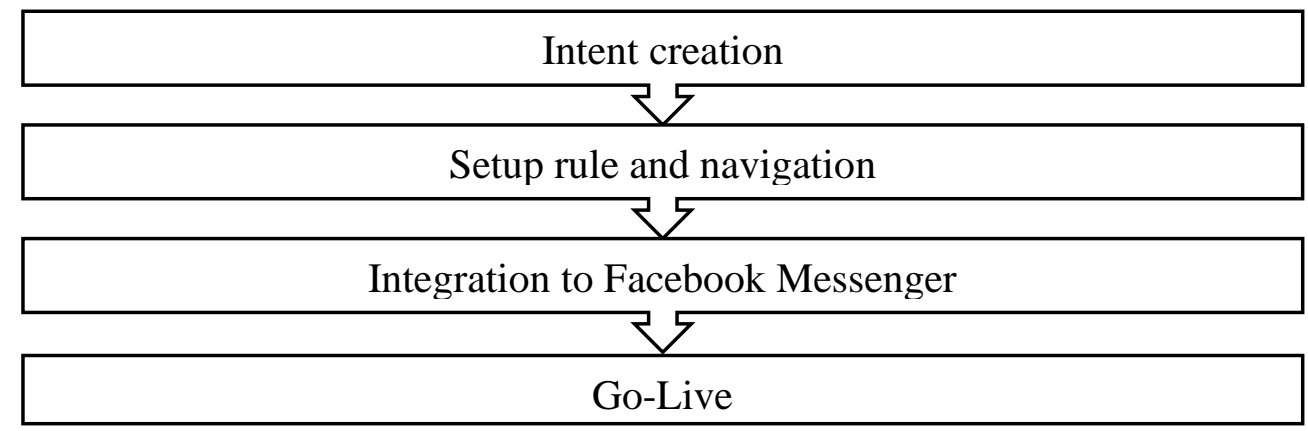

Figure 2: Development of Chatbot

\section{Research Outcome}

\section{Literature Survey}

From the reading and collection of literature, it was found that there are 7 dominant features that are favoured by the users as to be included for a good quality chatbot. These features are functionality, efficiency, humanity, effectiveness, technical satisfaction, as well as ethics. Table 3 describes the details for each category as obtained from the literature survey. 
Volume 6 Issue 22 (September 2021) PP. 88-102

DOI: $10.35631 / J I S T M .622008$

Table 3: Most Favoured Attributes for a Good Chatbot

\begin{tabular}{|c|c|c|}
\hline No. & Category & Quality Attributes \\
\hline 1 & Functionality & $\begin{array}{l}\text { - Interpret commands accurately (Morrissey and Kirakowski, } \\
\text { 2013) } \\
\text { - Flexible in interpreting knowledge (Cohen \& Lane, 2016). } \\
\text { - Number of services available in the chatbot (Eeuwen, } \\
\text { 2017). } \\
\text { - Suitability (ISO/IEC, 2001). } \\
\text { - Accuracy (ISO/IEC, 2001). } \\
\text { - Interoperability (ISO/IEC, 2001). } \\
\text { - Compliance (ISO/IEC, 2001). } \\
\text { - Security (ISO/IEC, 2001). }\end{array}$ \\
\hline 2 & Efficiency & $\begin{array}{l}\text { - } \quad \text { Ease of use (Candela, } 2018 \text { \& Duijst, 2018) } \\
\text { - } \quad \text { Quick replies vs free text (Duijst, 2018) } \\
\text { - } \quad \text { Available at all times (Wang et. al., 2018). } \\
\text { - } \quad \text { Accessibility (Duijst, 2018). } \\
\text { - } \quad \text { Time behavior (ISO/IEC, 2001). } \\
\text { - } \quad \text { Resource (ISO/IEC, 2001). } \\
\text { - } \quad \text { Utilization (ISO/IEC, 2001). } \\
\text { - } \quad \text { Strong to manipulation of data input by users (Saaty, 1990). }\end{array}$ \\
\hline 3 & Humanity & $\begin{array}{l}\text { - Realness of robot (Ramos, 2017) } \\
\text { - Create an enjoyable interaction (Morrissey \& Kirakowski, } \\
\text { 2013). } \\
\text { - Convey personalities (Morrissey \& Kirakowski, 2013) \& } \\
\text { (Kuligowska, 2015). } \\
\text { - Able to maintain the theme of the discussion (Smutny \& } \\
\text { Schreiberova, 2020). } \\
\text { - Able to respond to specific inquiry (Smutny \& } \\
\text { Schreiberova, 2020). } \\
\text { - Ability to mimic human personality (Saaty, 1990). } \\
\text { - Ability to provide correct response to user's request (Saaty, } \\
\text { 1990). }\end{array}$ \\
\hline 4 & Effectiveness & $\begin{array}{l}\text { - Construe statements and commands correctly (Saaty, } \\
\text { 1990). } \\
\text { - Linguistic precision (Saaty, 1990). } \\
\text { - Accurate task execution (Saaty, 1990). } \\
\text { - Consist of vast area of knowledge (Saaty, 1990). } \\
\text { - Ability to solve simple task request (Saaty, 1990). }\end{array}$ \\
\hline 5 & $\begin{array}{c}\text { Technical } \\
\text { satisfaction }\end{array}$ & $\begin{array}{l}\text { - Ability to express feeling (Saaty, 1990). } \\
\text { - Provide entertainment and enjoyment to user (Saaty, 1990). } \\
\text { - Conveying emotional information through tones and } \\
\text { expression (Saaty, 1990). }\end{array}$ \\
\hline 6 & Ethics & $\begin{array}{l}\text { - User's privacy protection (Saaty, 1990). } \\
\text { - Consistency (Saaty, 1990). } \\
\text { - Training the bot with knowledge on cultural and ethics } \\
\text { (Saaty, 1990). }\end{array}$ \\
\hline
\end{tabular}




\section{Pre-development}

A set of questionnaires has been prepared with two sections; chatbot evaluation survey (adapted from Griol \& Callejas, 2013), and satisfaction level of chatbot user (Brooke, 1996). Reliability testing has been carried out for these two sections. The first section, chatbot evaluation survey, with 10 questions distributed among 32 respondents, recorded an alpha coefficient value of 0.906 . For the second section, satisfaction level of chatbot recorded a value of 0.722 . Table 4 explains the values for the reliability testing. Table 5 describes the statements for Chatbot Evaluation Survey, and Table 6 describes the statements related to the satisfaction level of the chatbot's user.

Table 4: Reliability Testing

\begin{tabular}{lcc}
\hline \multicolumn{3}{c}{ Reliability Statistics } \\
\hline \multicolumn{1}{c}{ Section } & Cronbach's Alpha & N of Items \\
\hline Chabot Evaluation Survey & .906 & 10 \\
Satisfaction Level of Chatbot & .722 & 10 \\
\hline
\end{tabular}

Table 5: Section B: Chatbot Evaluation Survey (Griol \& Callejas, 2013)

\begin{tabular}{cl}
\hline No. & Statement (s) \\
\hline 1 & The system offers enough interactivity. \\
2 & The system is easy to use. \\
3 & It is easy to know what to do at each moment. \\
4 & The amount of information that is displayed on the screen is adequate. \\
5 & The arrangement of information on the screen is logical. \\
6 & The chatbot is helpful. \\
7 & The chatbot is interactive. \\
8 & The chatbot reacts in a consistent way. \\
9 & The chatbot complements the activities without distracting or interfering with them. \\
10 & The chatbot provides adequate non-verbal feedback. \\
\hline
\end{tabular}

Table 6: Section C: Satisfaction Level of Chatbot's User (Brooke, 1996)

\begin{tabular}{cl}
\hline No & Statement \\
\hline 1 & I think that I would like to use this system frequently. \\
2 & I found the system unnecessarily complex. \\
3 & I thought the system was easy to use. \\
4 & I think that I would need the support of a technical person to be able to use this system. \\
5 & I found the various functions in this system well integrated. \\
6 & I thought there were too many inconsistencies in this system. \\
7 & I would imagine most people would learn to use this system very quickly. \\
8 & I found the system very cumbersome to use. \\
9 & I felt very confident using the system. \\
10 & I needed to learn a lot of things before I could get going with this system.
\end{tabular}


Volume 6 Issue 22 (September 2021) PP. 88-102 DOI: 10.35631/JISTM.622008

Another section included in the pre-development stage questionnaire is about the attributes favoured by the user to have in any good chatbot. Figure 3 states the attributes chosen by the participants.

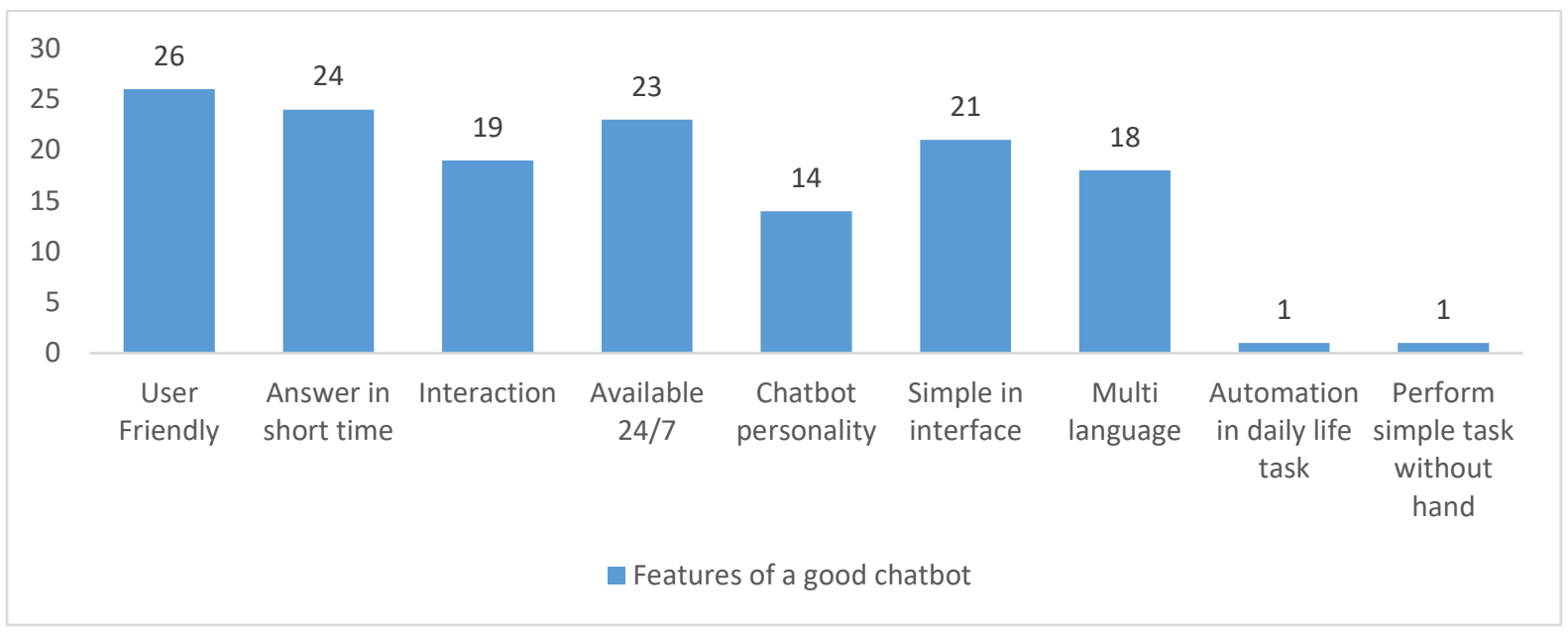

Figure 3: Attributes Favoured for A Good Chatbot

\section{Development of Chatbot}

The development of the chatbot started by selecting the platform which is Flow XO. It is an online platform that is equipped with pre-built templates for ease of use for new developers. Flow XO requires the developer to create an account in order to start building the bot. They also have other login options using Facebook or Google credentials. Figure 4 shows the main page of the log-in interface.

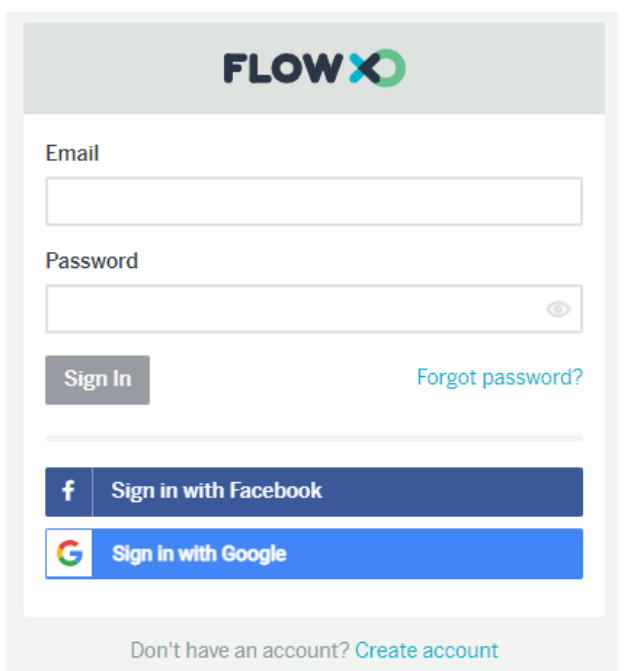

Figure 4: Log-in Page (from: flowxo.com) 
Volume 6 Issue 22 (September 2021) PP. 88-102 DOI: 10.35631/JISTM.622008

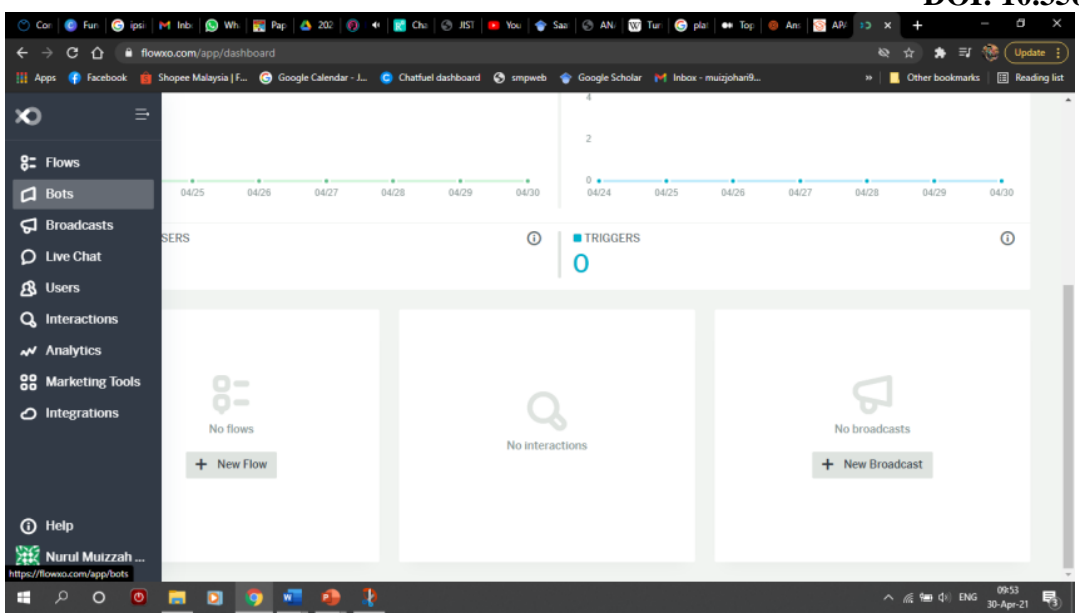

Figure 5: Dashboard Interface for Flow XO

Test \& Distribute

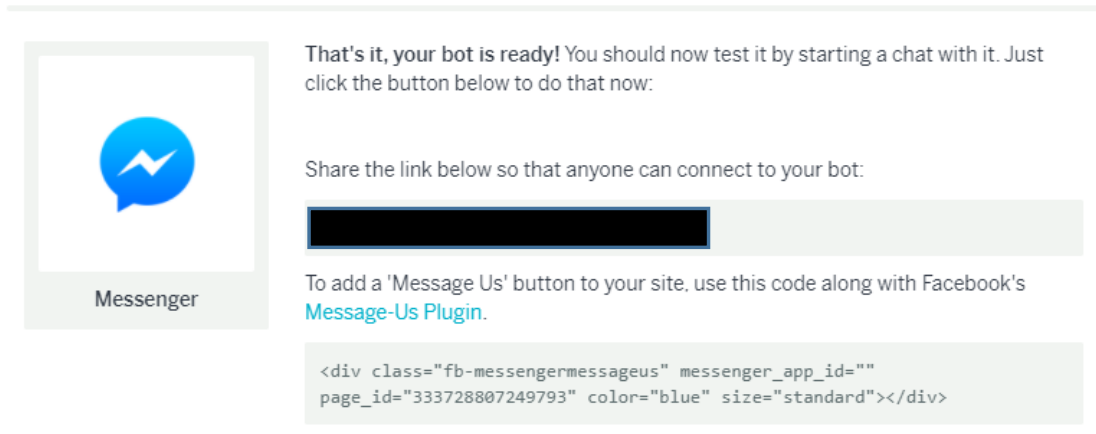

Figure 6: Integration with Facebook Messenger

After logged in to the account, developer will be directed to the platform's Dashboard as shown in Figure 5. All features available for the developer to start building are shown in the tab on the left-hand side. Integration to Facebook Messenger can be done right after registering the account. The developer needs to log into their Facebook account and link the bot to the desired landing page in Facebook to allow the bot to be embedded into the messenger setting. 


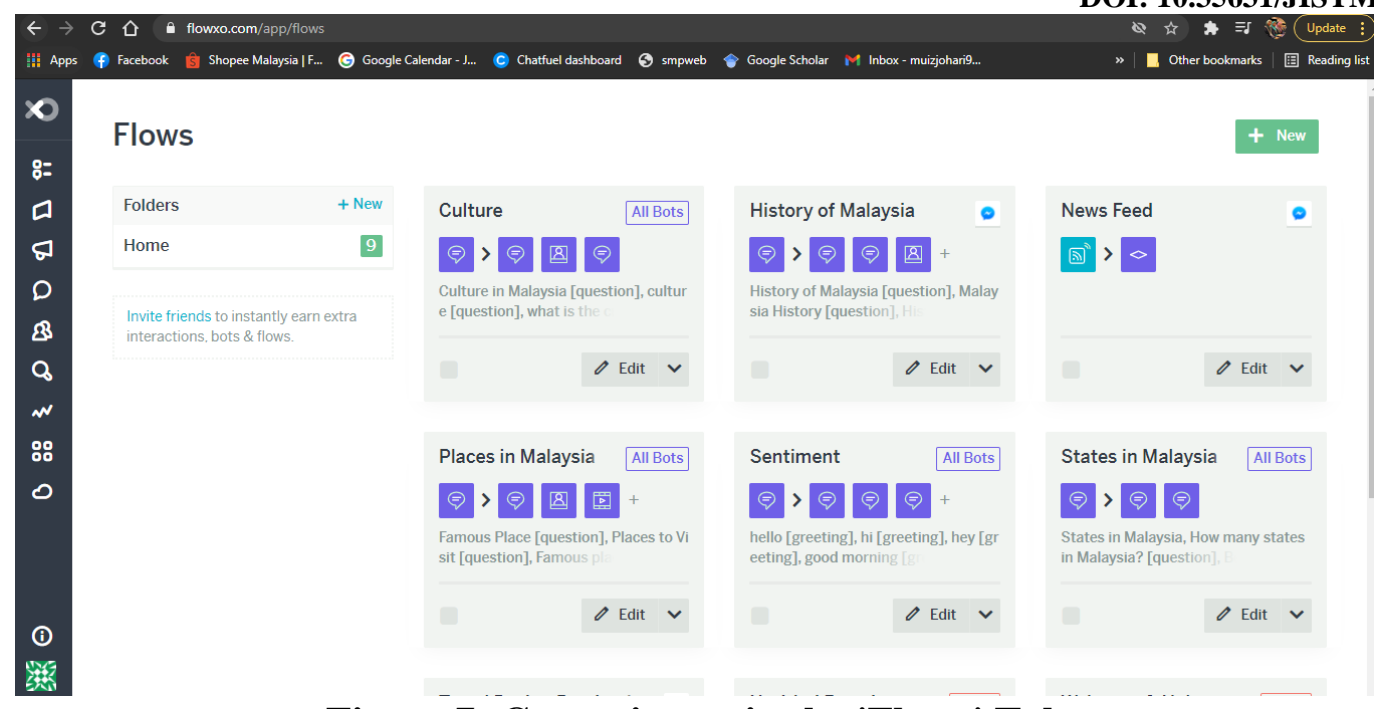

Figure 7: Create intent in the 'Flows' Tab

The creation of intent as the base knowledge for the bot can be done by using the features in the "Flows" tab as shown in Figure 7. Developer may also use the pre-built intents as a start to initiate the bot, and then add other intents to complete the objective of the bot creation. For this research, Tourism is chosen as the domain for the bot. Thus, all content will be based on tourism in Malaysia.

A creation list of intents with many possible questions or statements is crucial for the bot to interact smoothly with end users and respond with the answer set by the developer. Table 7 shows an example of the list for intent creation as knowledge base for the bot. Spelling mistakes, short words, unstructured phrases, as well as mixed language has been trained during intent creation to diversify the understanding of the chatbot to natural human conversation pattern.

Table 7: Example for List of Intent Use as Knowledge Base for The Bot

\begin{tabular}{|c|c|c|}
\hline Intent & Answer & Possible statement / question \\
\hline $\begin{array}{ll}\text { History of } \\
\text { Malaysia }\end{array}$ & $\begin{array}{l}\text { Malaysia, country of Southeast Asia, } \\
\text { lying just north of the Equator that is } \\
\text { composed of two noncontiguous } \\
\text { regions: Peninsular Malaysia } \\
\text { (Semenanjung Malaysia), also called } \\
\text { West Malaysia (Malaysia Barat), } \\
\text { which is on the Malay Peninsula, and } \\
\text { East Malaysia (Malaysia Timur), } \\
\text { which is on the island of Borneo. } \\
\text { Malaysia has achieved independent } \\
\text { on 31st August 1957. }\end{array}$ & $\begin{array}{l}\text { History of Malaysia [question] } \\
\text { Malaysia History [question] } \\
\text { History [question] } \\
\text { Origin of Malaysia [question] } \\
\text { How Malaysia is form? [question] } \\
\text { Where is Malaysia [question] } \\
\text { where's malaysia [question] } \\
\text { location [question] } \\
\text { locate malaysia [question] }\end{array}$ \\
\hline $\begin{array}{l}\text { Travel } \\
\text { during } \\
\text { pandemic }\end{array}$ & $\begin{array}{l}\text { Currently, there are some restrictions } \\
\text { to travel within and outside of } \\
\text { Malaysia due to the Covid } 19 \\
\text { pandemic outbreak. }\end{array}$ & $\begin{array}{l}\text { How to travel during pandemic } \\
\text { breakout? [question] } \\
\text { Is it safe to travel to now? [question] }\end{array}$ \\
\hline
\end{tabular}


However, you can still enjoy the breath-taking scenery of Malaysia. As long as all procedure stated by the government and Ministry of Health are being followed.
Culture in The largest ethnic groups in Malaysia Malaysia

are the Malays, Chinese and Indians. In Sabah and Sarawak, there are a myriad of indigenous ethnic groups with their own unique culture and heritage.

This multicultural context makes Malaysia a highly rich society, with diverse religions, foods, culture, and customs.
Volume 6 Issue 22 (September 2021) PP. 88-102 DOI: 10.35631/JISTM.622008

can we travel to Malaysia now?

[question]

domestic travel[question]

travel in malaysia[question]

SOP [question]

procedure needed for

travel[question]

where can travel during

covid19[question]

covid[question]

Culture in Malaysia [question]

culture [question]

what is the culture in Malaysia? [

question]

malaysian culture [question]

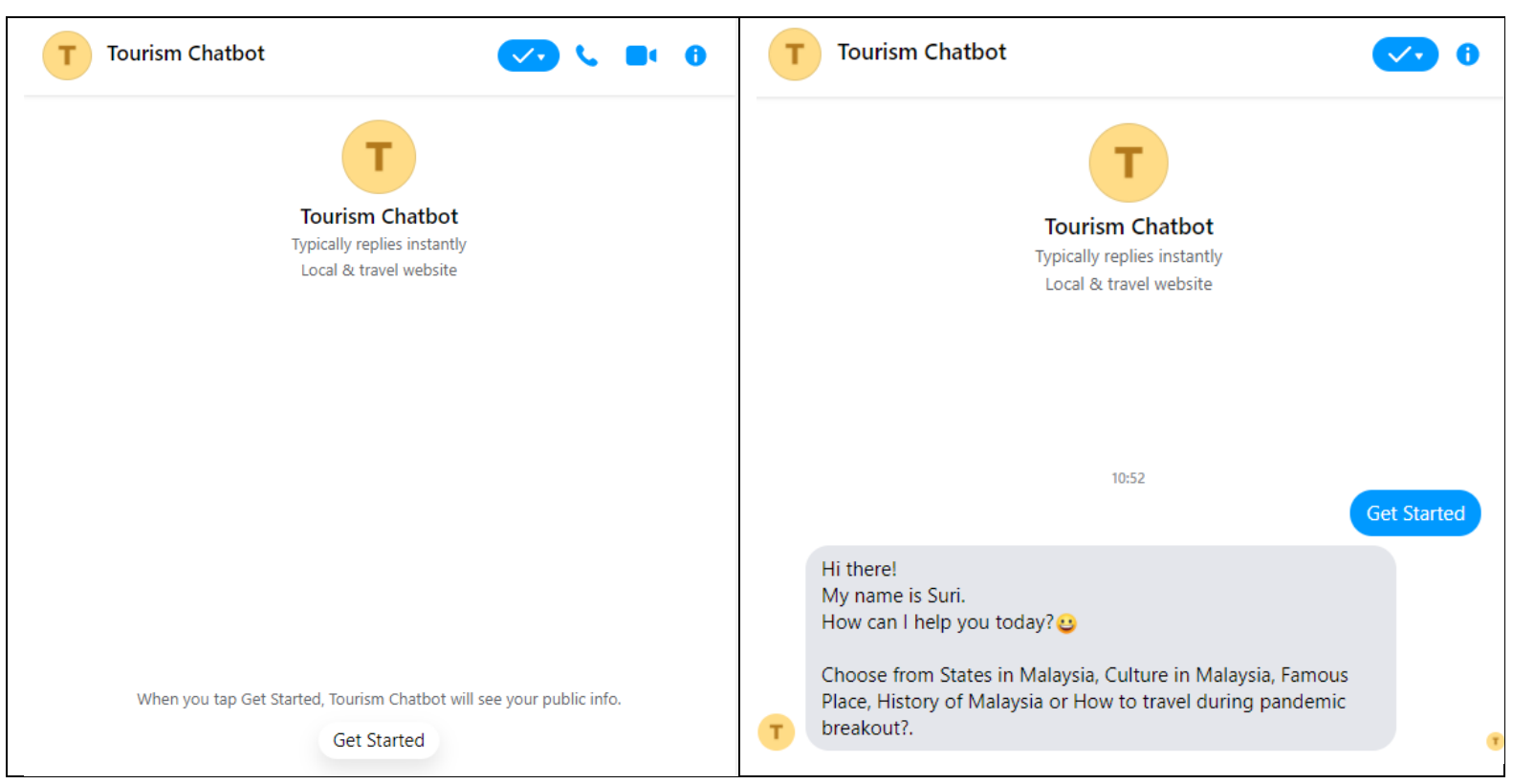

Figure 8: Chatbot in Facebook Messenger

To interact with the chatbot, user may use the button "Get Started" at the bottom of the messenger or they may start to input any keyword or sentence. The chatbot will greet the user and show the available menu to start the interaction, as shown in Figure 8. 


\section{Discussion and Conclusion}

The creation of the bot is based on the six features obtained from the pre-development phase: functionality, efficiency, humanity, effectiveness, technical satisfaction, and ethics. Table 8 summarizes the features available in the bot created using Flow XO with consideration from features gained in Phase I, pre-development.

Table 8: Features from Literature Survey vs. Flow XO

\begin{tabular}{ll}
\hline Category & New chatbot description \\
Functionality & $\begin{array}{l}\text { Facebook Messenger is commonly used by all Facebook users. Thus, } \\
\text { they are well versed with the function. } \\
\text { Efficiency }\end{array}$ \\
$\begin{array}{l}\text { User is able to input any word or sentence and the bot is still able to } \\
\text { respond in a short time with a related answer to the inquiry. } \\
\text { The language used for the bot's conversation is simplified to non-formal } \\
\text { to reflect friendly personality. }\end{array}$ \\
Effectiveness & $\begin{array}{l}\text { A wide array of knowledge set by the developer allows the bot to learn } \\
\text { and respond to any question given by the user. }\end{array}$ \\
Technical & $\begin{array}{l}\text { The bot uses simple words to greet the user at the beginning and to end } \\
\text { the conversation. }\end{array}$ \\
satisfaction & $\begin{array}{l}\text { The pattern of conversation is within the theme until the user requests to } \\
\text { change. } \\
\text { The bot is trained with ethics by the developer through intent creation by } \\
\text { taking point on the culture and ethics. }\end{array}$ \\
\hline
\end{tabular}

However, there is a limitation to creating chatbots using the Flow XO platform, as it is only limited to a certain number of conversations. This issue restricts developers from creating as many intents as possible in order to train the bot. The work around provided by the platform to overcome this limitation in intents, is by providing a subscription-based option for further usage. Once subscribed, the developers are able to utilize up to 15 flows or intents, which broadens the opportunity to train the bot effectively.

The chatbot developed as a result of this paper is under development stage. This condition indicates that this system is open to amendments from time to time accordingly. Once the planned architecture of the chatbot has been completed, there will be an extensive research in the future to evaluate the features of the newly developed chatbot. The measurement will be in the form of user experience while using the services provided by the chatbot using structured questionnaires, with validation from field experts as well as from targeted users.

\section{References}

Aishwarya, B., Vijay, G., \& Sachin, D. (2017). Chatbot for Business Organization: Utility, Processes and Significance. International Journal of Scientific Research in Computer Science, Engineering, and Information Technology,2(6), 872-881.

Barker, S. (2021, March 18). Top Challenges and Benefits of AI Chatbots. Shane Barker. https://shanebarker.com/blog/challenges-and-benefits-of-ai-chatbots/

Beale, R. \& Creed, C. (2009). Affective interaction: How emotional agents affect users. International Journal of Human-Computer Stuides, 67, 755-776.

Belfin, R. V., Shobana, A. J., Manilal, M., Mathew, A. A., \& Babu, B. (2019, March). A graph based chatbot for cancer patients. In 2019 5th International Conference on Advanced Computing \& Communication Systems (ICACCS) (pp. 717-721). IEEE. 
Brooke, J. (1996). SUS: A Quick and Dirty Usability Scale. In. P.W. Jordan, B. Thomas, B. A. Weerdmeeser \& I.L. McClelland (Eds.). Usability Evaluation in Industry. London: Taylor \& Francis

Candela, E. (2018). Consumer's perception and attitude towards chatbots adoption. A focus on the Italian market. Aalborg University.

Cohen, D., \& Lane, I. (2016, february). An oral exam for measuring a dialog's system's capabilities. In Porceedings of the Thirtieth AAAI Conference On Artificial Intelligence. (pp.835 - 841). AAAI Press.

Eeuwen, M. (2017). Mobile conversation commerce: messenger chatbots as the next interface between businesses and consumers (Master's Thesis, University of Twenty) Retrieved on April 20, 2021 from http://essay.utwente.nl/71706/1/van\%20Eeuwen_MA_BMS.pdf

Folstad, A., \& Skjuve, M. (2019). Chatbots for customer service: user experience and motivation. In Proceeding of the International Conference on Conversational User Interface-CUI, ACM, New York.

Griol, D., \& Callejas, Z. (2013). An arechitecture to develop multimodal educative applications with chatbots. International Journal of Advanced Robotic Systems, 10(3), 175.

ISO/IEC (2001). ISO/IEC 9126. Software Engineering - Product quality. ISO/IEC.

Kuligowska, K. (2015). Commercial chatbot: performance evaluation, usability metrics and quality standards of embodied conversational agents. Professionals Center for Business Research, 2.

Meerschman, H. \& Verkeyn, J. (2019). Towards A Better Understanding of Service Quality Attributes of a Chatbot (Master Dissertation). Retrieved from https://libstore.ugent.be/fulltxt/RUG01/002/784/375/RUG01002784375_2019_0001_ AC.pdf

Monisha, S. G. (2018, October 9). 4 Biggest Challenges in Chatbot Development and How to Avoid them. Software Technology Insights. https://insights.daffodilsw.com/blog/4biggest-challenges-in-chatbot-development-and-how-to-avoid-them

Morrissey, K., \& Kirakowski, J. (2013, July). 'Realness' in Chatbots: Establishing Quantifiable Criteria. In International Conference on Human-Computer Interaction. (pp. 87-96). Springer Berlin Heidelberg.

Patil, P. (2020, July 17). Top 30 Most Popular Platforms To Build Chatbots | Chatbots Journal. Chatbots Journal. https://chatbotsjournal.com/top-30-powerful-and-best-platforms-tobuild-chatbots-bf413419d584

Rarhi, K., Bhattacharya, A., Mishra, A., \& Mandal, K. (2017). Automated medical chatbot. Available at SSRN 3090881

Rieke, T. D. (2018). The relationship between motives for using a Chatbot and satisfaction with Chatbot characteristics in the Portuguese Millenial population: an exploratory study. Thesis. Faculty of Economics. Universitiy of Porto.

Saaty, T. L. (1990). How to make decision: The analytic heirarchy process. European Journal of Operation Research,48 (1), 9-26.

Smutny, P. \& Schreiberova, P. (2020). Chatbots for learning: A review of educational chatbots for the Facebook Messenger. Computers \& Education, 151,106832.

Tamayo, P. A., Herrero, A., Martín, J., Navarro, C., \& Tránchez, J. M. (2020). Design of a Chatbot as a Distance Learning Assistant. Open Praxis, 12(1), 145-153.

Thompson, S. A. (2002). "Object Complements" and conversation towards a realistic account. Stuidies in Language , International Journal sponsored by the Foundation " Foundations of Language”,26(1), 125-163.

Copyright $\odot$ GLOBAL ACADEMIC EXCELLENCE (M) SDN BHD - All rights reserved 
Volume 6 Issue 22 (September 2021) PP. 88-102 DOI: 10.35631/JISTM.622008

Ukpabi, D. C., Aslam, B., \& Karjaluoto, H. (2019). Chatbot adoption in tourism services: A conceptual exploration. In Robots, artificial intelligence, and service automation in travel, tourism and hospitality. Emerald Publishing Limited.

Weizenbaum, J. (1996). ELIZA - a computer program for the study of natural language communication between man and machine. Communication of the AC,9(1), 36-45.

Winkler, R., \& Soellner, M. (2018). Unleashing the potential of chatbots in education: A stateof-the-art analysis. 